\title{
Excited states of beryllium atom from explicitly correlated wave functions.
}

\author{
F.J. Gálvez and E. Buendía \\ Departamento de Física Moderna, Facultad de Ciencias, \\ Universidad de Granada, E-18071 Granada, Spain \\ A. Sarsa \\ International School for Advanced Studies, SISSA \\ Via Beirut 2/4, I-34014 Trieste, Italy
}

\begin{abstract}
A study of the first excited states of beryllium atom starting from explicitly correlated wave functions is carried out. Several properties are obtained and discussed focusing on the analysis of the Hund's rules in terms of the single-particle and electron pair intracule and extracule densities. A systematic study of the differences on the electronic distributions of the singlet and triplet states is carried out. The trial wave function used to describe the different bound states consists of a generalized Jastrowtype correlation factor times a configuration interaction model wave function. This model wave function has been fixed by using a generalization of the optimized effective potential method to deal with multiconfiguration wave functions. The optimization of the wave function and the calculation of the different quantities is carried out by means of the Variational Monte Carlo method.
\end{abstract}




\section{INTRODUCTION}

Most of the studies on the excited states of the beryllium atom have been focused on the attainment of the binding energy. They have been mainly carried out by using extensive Configuration Interaction type wave functions obtaining very accurate results [1]. Recently very precise values for the energy and related properties such as the electron affinity or mass shifts have been calculated by making use of the Quantum Monte Carlo method [2] and by using large expansions of exponentially correlated Gaussian functions [3]. However the knowledge of other interesting properties of these states is much more scarce. For example, one and two body electron densities, which provide valuable information on the structure and dynamics of the system are not fully characterized. This is due to the technical difficulties appearing in their calculation. These densities play an important role in the understanding and in the interpretation of some interesting features of the electronic structure of atoms such as the Coulomb hole and the Hund's rule [4]-[7]. This rule states that if two states arise from the same configuration, the state having the higher spin will have the lower energy. For those states with the same spin value, the most bound one is that with the higher value of the orbital angular momentum. Contrary to qualitative arguments, the electron-electron repulsion is higher for the triplet state, and is the deeper electron-nuclear attraction energy value of the triplet state the responsible for the lower triplet energy. This has been shown for some excited states of Helium-like systems by using Hylleraas type wave functions 8 , for the ${ }^{1} P \_3 P$ terms arising from the $2 s-2 p$ configuration in beryllium, by using both Hartree-Fock and Configuration Interaction wave functions [9]-[14], and also for some other atomic and molecular systems within the Hartree-Fock framework [15, 16].

The aim of this work is to extend previous studies to the first excited states of the beryllium atom with symmetry ${ }^{1} S,{ }^{3} S,{ }^{1} P,{ }^{3} P,{ }^{1} D$ and ${ }^{3} D$ by using explicitly correlated wave functions. Starting from those wave functions the energy and some different one- and twobody position and momentum properties as well as the single-particle density and both the interelectronic (intracule) and the center of mass (extracule) two-body densities in position space have been obtained. Explicitly correlated wave functions fulfill some analytically known properties of the densities, as for example the correct short range behavior and cusp conditions which become very important in describing correctly the Coulomb hole. These distribution functions provide a picture of the spatial arrangement of the electrons and will be analyzed in order to get insight on the correlation effects and on the Hund's rule.

The single-particle density is defined as

$$
\rho(\vec{r})=\int \sum_{i=1}^{N} \delta\left[\vec{r}-\vec{r}_{i}\right] \frac{\left|\Psi\left(\vec{r}_{1}, \vec{r}_{2}, \ldots, \vec{r}_{N}\right)\right|^{2}}{\langle\Psi \mid \Psi\rangle} d \vec{r}_{1} d \vec{r}_{2} \ldots d \vec{r}_{N}
$$

the interelectronic or intracule density is given by 


$$
I\left(\vec{r}_{12}\right)=\int \sum_{i>j=1}^{N} \delta\left[\vec{r}_{12}-\left(\vec{r}_{i}-\vec{r}_{j}\right)\right] \frac{\left|\Psi\left(\vec{r}_{1}, \vec{r}_{2}, \ldots, \vec{r}_{N}\right)\right|^{2}}{\langle\Psi \mid \Psi\rangle} d \vec{r}_{1} d \vec{r}_{2} \ldots d \vec{r}_{N}
$$

and, finally, the center of mass or extracule density can be calculated as

$$
E(\vec{R})=\int \sum_{i>j=1}^{N} \delta\left[\vec{R}-\left(\vec{r}_{i}+\vec{r}_{j}\right) / 2\right] \frac{\left|\Psi\left(\vec{r}_{1}, \vec{r}_{2}, \ldots, \vec{r}_{N}\right)\right|^{2}}{\langle\Psi \mid \Psi\rangle} d \vec{r}_{1} d \vec{r}_{2} \ldots d \vec{r}_{N}
$$

The corresponding spherically averaged densities are labelled as $\rho(r), h\left(r_{12}\right)$ and $d(R)$, respectively, and represent the probability density functions for the electron-nucleus distance, $r$, the interelectronic distance, $r_{12}$, and the center of mass distance, $R$. The single-particle density is normalized to the number of electrons, $N$, and both the intracule and the extracule densities to the number of electron pairs, $N(N-1) / 2$. The value of the intracule and extracule densities at the origin give us the probability of two electrons to be at the same position and at opposite positions with respect to the nucleus, respectively.

The wave functions used in this work include explicitly the electronic correlations by means of a Jastrow-type factor. Nondynamic effects are also taken into account by using a multi configuration model wave function where the most important substitutions are included. This parameterization has shown to provide accurate results for the ground state of the isoelectronic series of the beryllium atom [17]. The calculation of the different expectation values has been carried out by means of the Variational Monte Carlo method [18].

The structure of this work is as follows. In Sec. II the trial wave function is shown in detail. The results are presented and discussed in Sec. III. The conclusions of this work can be found in Sec. IV. Atomic units are used throughout unless otherwise indicated.

\section{WAVE FUNCTION}

The correlated trial wave function, $\Psi$, used in this work is the product of a symmetric correlation factor, $F$, which includes the dynamic correlation among the electrons, times a model wave function, $\Phi$, that provides the correct properties of the exact wave function such as the spin and the angular momentum of the atom, and is antisymmetric in the electronic coordinates.

$$
\Psi=F \Phi
$$

For the correlation factor we use the form of Boys and Handy [19 with the prescription proposed by Schmidt and Moskowitz [20]. In particular we have worked with 17 variational non-linear parameters in the correlation factor which include electron-nucleus, electronelectron and electron-electron-nucleus correlations. 
In order to get the different bound states of a given $L, S$ symmetry we make use of the well known Hylleraas-Undheim theorem. The hamiltonian is diagonalized in a set of $m$ linear independent trial functions with the proper symmetry and then the eigenvalues constitute upper bounds to the first $m$ bound states. The trial wave functions are linear combinations of Slater determinants built starting from the single particle configurations $1 s^{2} n l n^{\prime} l^{\prime}$, with $n, n^{\prime}=2,3$ and $l, l^{\prime}=s, p, d$, coupled to the total orbital angular momentum and spin of the term under study times the Jastrow correlation factor aforementioned. For the ${ }^{1} D$ state we have also considered the configuration $1 s^{2} 2 s 4 d$.

The model wave function has been fixed within the optimized effective potential framework. This is a mean field approximation to the many electron problem based on finding the best local one-body potential minimizing the total energy, which is expressed as an orbital functional as in the Hartree-Fock approach. This method was first proposed by Slater [21] as a simplification of the Hartree-Fock equations and further developed and applied to atomic problems by Talman and coworkers [22, 23]. The trial wave function is a Slater determinant with single-particle wave functions calculated from a certain effective potential that is assumed to be central. The minimum condition on the effective potential leads to a set of non-linear coupled integro-differential equations involving the potential and the orbitals. They can be solved by means of a self-consistent procedure just like in the Hartree-Fock method by using either numerical-grid techniques [22, 23] or by expanding the effective potential in a finite basis set that guarantees the fulfillment of the correct asymptotic behavior 24.

In this work we have generalized this method to deal with multi configuration wave functions instead a single Slater determinant

$$
\Phi_{m c}=\sum_{k} C_{k} \phi_{k}
$$

where $\phi_{k}$ are Slater determinants built with the eigenfunctions of the effective potential, which has been parameterized in the present work as follows

$$
V(r)=\frac{1}{r}\left\{(Z-N+1)+(N-1) \sum_{k} a_{k} e^{-b_{k} r}\right\}
$$

where $Z, N$ are the nuclear charge and the number of electrons, respectively, and $\left\{a_{k}, b_{k}\right\}$ are variational parameters with the constraint $\sum a_{k}=1$ to match the exact short range behavior of the potential. It also reproduces the exact long range behavior.

Within this scheme, given some particular values of the parameters $\left\{a_{k}, b_{k}\right\}$ the one-body Schrödinger equation defined by the effective potential is solved. The orbitals obtained by solving this single particle Schrödinger equation are used to write down the Slater determinants appearing in the multi configuration expansion of the trial wave function of Eq.(5)). 
Then the total energy is calculated as the expectation value of the hamiltonian in that trial wave function. The linear coefficients $C_{k}$ of the configuration interaction expansion of the model wave function are also obtained in this step. The optimum set of parameters of the effective potential is fixed by imposing the minimum condition on the total energy. Finally it is worth to point out that the one-body problem has been solved here by expanding the radial part of the single particle wave functions in terms of Slater functions as in the Roothaan-Hartree-Fock method.

The purpose of the present work is not to obtain the best description of the excited states of the beryllium atom within the effective potential framework. It constitutes only a previous step that produces the model wave functions used along with the correlation Jastrow factor. Therefore full convergence in the number of free parameters of the effective potential has not been pursued. Instead a certain compromise between the number of free parameters and the accuracy at the effective potential framework has been found. For example when using the single configuration $1 s^{2} 2 s^{2}$ to describe the ground state of the Be atom, the effective potential wave function used here provides an energy of -14.57235 au to be compared with the optimized effective potential results -14.57245 au and -14.57256 au reported in [23] and 24] respectively and with the Hartree-Fock value $-14.57302 \mathrm{au}$. In the case of a multi configuration wave function built from the linear combination of the configurations $1 s^{2} 2 s^{2}$ and $1 s^{2} 2 p^{2}$ that takes into account the near degeneracy effect and constitutes the complete active space wave function for this atom, the optimized effective potential energy obtained by us is -14.61556 au while the exact Multi Configuration Hartree Fock (MCHF) value with these two configurations is -14.61685 au [25]. For the sake of completeness we mention here that the energy when a Jastrow correlation factor is used along with the effective potential model function obtained here is $-14.6647(1)$ au that coincides with the value of [26] where a MCHF was used as model function, to be compared with the estimated exact energy -14.66736 au 25.

The way in which the different calculations have been carried out is the following. For any given a state, for example the $2 s 3 s^{-1} S$, the set of configurations that are thought to be the most important is selected, $1 s^{2} 2 s^{2}, 1 s^{2} 2 p^{2}, 1 s^{2} 2 s 3 s$ and $1 s^{2} 2 p 3 p$ in our example. With them the model wave function, $\Phi_{m c}$, is fixed within the effective potential framework. The diagonalization gives rise to a set of orthogonal and non correlated vectors (four in our example). A new correlated basis set is built up by multiplying each one of the noncorrelated vectors by the correlation factor, $F$, which depends on 17 variational non-linear parameters. In this new basis set, which is non-orthogonal, we diagonalize the atomic hamiltonian, obtaining the eigenvectors along their corresponding eigenvalues, which are upper bounds to the four first states of ${ }^{1} S$-type. Because we are interested in the excited $2 s 3 s-{ }^{1} S$ state, we optimize the non-linear parameters of the correlation factor by minimizing the second eigenvalue. The minimization has not been carried out on the variance but on the energy, that has shown to provide not only more accurate upper bounds [27] but also 
a better description of other properties [26]. The final wave function, $\Psi_{c m c}$, is written as a correlated basis function expansion, that in our example is

$$
\Psi_{c m c}\left[2 s 3 s ;^{1} S\right]=F\left(d_{1} \phi\left[2 s^{2} ;^{1} S\right]+d_{2} \phi\left[2 p^{2} ;^{1} S\right]+d_{3} \phi\left[2 s 3 s ;^{1} S+d_{4} \phi\left[2 p 3 p ;^{1} S\right]\right)\right.
$$

where the core $1 s^{2}$ is implicit in each one of the configurations. The new coefficients $d_{k}$ are obtained in the diagonalization of the Hamiltonian in the correlated basis, and the different matrix elements of the hamiltonian in this basis set have been calculated by using the Monte Carlo algorithm.

\section{RESULTS}

The use of a large number of configurations increases greatly the computer time and also the numerical errors in the diagonalization. Therefore we have to limit ourselves to consider a number of configurations as small as possible. Thus, for each one of the states studied, a given configuration has been selected if the difference between the energies obtained with and without it is clearly greater than the statistical error in the calculation, once the correlation factor has been included. In some cases these configurations do not coincide with those most important if the correlation factor, $F$, is not considered. The best wave functions obtained in this work recover more than $90 \%$ of the correlation energy. The correlation energy has been traditionally defined in quantum chemistry calculations as the difference between the exact (non-relativistic, infinite nuclear mass) and the Hartree-Fock energies. However for some of the states studied in this work the Hartree-Fock method does not provide an upper bound on the energy. This problem is handled here by defining the correlation energy as the difference between the exact non relativistic energy and that obtained with the best model wave function $\Phi_{m c}$.

We have studied the first ten excited states of the beryllium atom, five singlets and five triplets. The configurations used to study each one of the states considered are shown in Table I. In general, configuration mixing is much more important for singlet than for triplet states. In this table we show the excitation energy, in $e V$, obtained for each one of the states studied. The non-correlated energy obtained from $\Phi_{m c}$ by using these configurations as well as the correlated energy obtained from $\Psi_{c m c}$ are compared with the estimated exact one [28]. In parenthesis we show the statistical error in the Monte Carlo calculation and, in brackets, the percentage of correlation energy recovered. For triplet states the difference between the energy obtained in this work and the exact one oscillates around $0.07 \mathrm{eV}$, except for the $2 s 3 p^{3} \mathrm{P}$ for which is $0.1 \mathrm{eV}$. For singlet states this difference goes from 0.1 to $0.14 \mathrm{eV}$. The greatest difference is $0.142 \mathrm{eV}$, i.e. 0.005 hartree, and corresponds to the state $2 s 3 p-{ }^{1} \mathrm{P}$. In general the contribution to a given singlet state from other configurations than the principal one is much more important than for a triplet state. As a consequence, the energy obtained for singlet states within a subspace given is, in general, of a poorer quality than for triplet states. 
In Tables II, III and IV we show the results obtained for the moments of the single-particle and the electron pair intracule and extracule densities, respectively, starting from the correlated wave function $\Psi_{c m c}$. We give the expectation values $\left\langle t^{n}\right\rangle$, where $t=r, r_{12}$ and $R$, respectively, and $n=-2,-1,1,2$ and 3 . We have also calculated the value of the singleparticle and of both, the intracule and the extracule, densities at the origin. In doing so the following relation has been used

$$
\chi(0)=-\frac{1}{2 \pi} \sum_{i>j} \int Q \Psi^{2}(x) \frac{1}{Q} \frac{1}{\Psi(x) t^{2}} \frac{\partial \Psi(x)}{\partial t} d x
$$

for the single-particle density $(\chi=\rho, t=r)$ [29] and for the intracule $\left(\chi=h, t=r_{12}\right)$ and the extracule $(\chi=d, t=R)$ densities [30]. These expressions allow one to obtain a local property of the corresponding density in terms of the wave function evaluated in the whole domain. In order to calculate all these expectation values we have used $\left|\Psi_{c m c}\right|^{2}$ as distribution function, except for $h(0)$ and $\left\langle r_{12}^{-2}\right\rangle$ for which we have used $Q\left|\Psi_{c m c}\right|^{2}$, with $Q=\sum_{i<j} 1 / r_{i j}^{2}$, which provides more accurate results for those expectation values.

To study the quality of the results in Tables II and III we compare the results obtained for the ground state with those than can be considered as exact obtained also from an explicitly correlated wave function [31], and with those obtained in [26] where a correlated wave function similar to $\Psi_{c m c}$ but with a MCHF model wave function was used instead. The results obtained in this work are of a great quality, improving those reported in [26]. In Tables III-IV we also included the results recently obtained for the ground state intracule and extracule densities of beryllium starting from a MCHF wave function 32. Despite these last results are unaffected of statistical errors and are very precise in a numerical sense, they are not as accurate as ours at low interelectronic distances which is the region where electronic correlations are more important. That shows the validity of our techniques and our wave function that, although approximated, accounts for the most relevant physical correlation mechanisms. In Tables III-IV we also include the HF results obtained for the moments of both the intracule and extracule densities of the $2 s 2 p-{ }^{1} P$ and $2 s 2 p-{ }^{3} P$ states [14] and some values obtained for the intracule density of those same states starting from a multi configurational wave function [12].

To study the effects of electronic correlations we have considered those states for which the single configuration Hartree-Fock method can be applied to obtain upper bounds to the exact non-relativistic energy of the corresponding state. These states are $2 s 2 p-{ }^{3} \mathrm{P}, 2 s 2 p-{ }^{1} \mathrm{P}$, $2 s 3 s{ }^{3} \mathrm{~S}, 2 p^{2}-^{1} \mathrm{D}, 2 p^{2}{ }^{3} \mathrm{P}$, and $2 s 3 d{ }^{-3} \mathrm{D}$, i.e. the most bounded state with a $L, S$ given, except the $2 p^{2}{ }^{3} \mathrm{P}$ state because at a Hartree Fock level this state is orthogonal to the $2 s 3 p-{ }^{3} \mathrm{P}$ one. For all these states we have worked with a monoconfigurational wave function calculated from the optimized effective potential approach, that it is very similar to the Hartree-Fock one. For some other states it is necessary to work with an interaction configuration wave 
function that includes partially correlation effects.

The differences found between correlated and Hartree-Fock results are much more important for singlet than for triplet states. To analyze systematically the different correlation mechanism included by the trial function we have calculated the following difference functions

$$
\begin{aligned}
f_{c m c-s c}(t) & =4 \pi t^{2}\left[f_{c m c}(t)-f_{s c}(t)\right] \\
f_{m c-s c}(t) & =4 \pi t^{2}\left[f_{m c}(t)-f_{s c}(t)\right] \\
f_{c m c-m c}(t) & =4 \pi t^{2}\left[f_{c m c}(t)-f_{m c}(t)\right]
\end{aligned}
$$

where $f$ and $t$ stand for $R$ and $r, H$ and $r_{12}$, and $D$ and $R$, respectively. The $f_{c m c}$ function is the corresponding density calculated from the best correlated wave function obtained in this work, $\Psi_{c m c}, f_{m c}$ correspond to a multiconfiguration wave function without Jastrow correlation factor, $\Phi_{m c}$, and finally $f_{s c}$ is the density calculated from the single configuration self consistent approach wave function. The $f_{c m c-s c}(t)$ difference functions account for the full effect of the electronic correlations on the densities, the $f_{m c-s c}(t)$ give us insight on the effects of the configuration mixing on the electronic distribution and finally $f_{c m c-m c}$ give us information on the importance of the Jastrow factor.

In Figure 1 we show the results obtained for $f_{m c-s c}$ and $f_{c m c-s c}$, for the $2 s 2 p-{ }^{1} P$ and $2 s 2 p-{ }^{3} P$ states which are representative for those results obtained for singlet and triplet states. The same is shown in Figure 2 for the $f_{c m c-m c}$ difference functions. Several are the effects that can be noticed:

i) The big magnitude of the several difference functions for singlet states as compared with those for triplet ones show that the monoconfigurational self consistent wave function is not adequate for describing singlet states and a multiconfigurational wave function is necessary to include those aspects more relevant in the structure of these states.

ii) For singlet states, the medium and long range behavior of the three difference functions is well described by the model wave function, $\Phi_{m c}$. However short range correlations, which are explicitly included in the correlation factor, $F$, are necessary to reproduce the Coulomb hole in $h\left(r_{12}\right)$ as well as the short $R$ behavior of the extracule density.

iii) For triplet states the conclusions are not clear: the difference functions $f_{m c-s c}$ are quite flat, except for the extracule function, although they follow the correct trend for large values of the variable and then they include large range correlations. The functions $f_{c m c-s c}$ show a great amount of structure for all the three densities. That indicates that for triplet states the interaction configuration wave function is not so important as in the case of singlet states. 
iv) As can be seen in Figure 2, the effect of the correlation factor on the three difference functions is similar for triplet and singlet states for short and medium distances, indicating that the correlation factor is nearly independent of the total spin of the system. The differences between singlet and triplet become more important at larger distances.

v) The main effects of the electronic correlations take place at low values of the interparticle distance in the intracule density, the so called Coulomb hole. The structure of this Coulomb hole is practically the same for all the states considered in this work.

A comparative study of the states arising from the same configuration shows that singlet states are more extended in space than triplet ones. This is due to the outermost electron, as can be checked by comparing the moments of positive order of singlet and triplet states in Tables II and III. This fact is particularly relevant for the $2 s 2 p, 2 s 3 d$ and $2 s 3 s$ configurations and specially important in the $2 p^{2}$ configuration. The $2 s 3 p$ configuration is the case where this effect is less important. We have verified that the Hartree-Fock approximation, although gives a qualitatively correct picture of this, provides values that overestimate this effect. Because of this more diffuse character of the electron cloud of the singlet as compared with the triplet one could think that both electron-electron and electron-nucleus interactions are stronger in the latter. However this does not hold in general, especially for high lying configurations. For example the electron-electron repulsion energy, $V_{e e}=\left\langle r_{12}^{-1}\right\rangle$, is higher for singlet than for triplet states in the configurations $2 s 3 p$ and $2 s 3 d$. Besides, the electronnucleus attraction energy, $V_{e n}=-Z\left\langle r^{-1}\right\rangle$ is slightly higher for the singlet state than for the triplet one in the configuration $2 s 3 p$.

The Hund's rule is satisfied for all the configurations studied in this work except for the $2 p^{2}$ one. In order to shed light over this fact we have analyzed the balance between the nuclear attraction $V_{e n}=-Z\left\langle r^{-1}\right\rangle$ and electron-electron repulsion $V_{e e}=\left\langle r_{12}^{-1}\right\rangle$ energies. For the $2 s 2 p$ and $2 s 3 s$ configurations the lower energy of the triplet is due to a tighter nuclear attraction that compensates the electron-electron repulsion that is also stronger in the triplet. This same interpretation has been given previously for other systems as helium-like ions [6] studied by means of correlated wave functions and some states of the atoms $\mathrm{C}, \mathrm{N}, \mathrm{O}, \mathrm{Si}, \mathrm{S}$ and others [15] studied by using self consistent type wave functions. This is the opposite to the traditional interpretation of the Hund's rule based on the assumption that the repulsion is smaller in the triplet state. We have found that the only configuration for which this traditional interpretation holds is the $2 s 3 p$ one, that presents a stronger repulsion in the singlet that compensates the effect of the nuclear attraction that is slightly bigger also for the singlet. In the case of the $2 s 3 d$ configuration both the nuclear attraction and the electronic repulsion tend to make the triplet more bounded than the singlet. Finally, the only configuration that does not verify the Hund's rule is the $2 p^{2}$ one. This is due to the stronger electronic repulsion of the triplet that beats the bigger nuclear attraction of this state as compared to the singlet. 
A comparative study of the singlet-triplet single-particle, intracule and extracule densities can be seen in Figure 3 where we show the difference functions $4 \pi t^{2}\left[f_{1}(t)-f_{3}(t)\right]$ where $t$ stands for $r, r_{12}$ and $R$, and $f_{1}\left(f_{3}\right)$ for the singlet (triplet) single-particle, intracule and extracule densities. In Figure 3 (top) we show the differences for the single-particle density. At very short distances appreciable differences are observed only for the $2 p^{2}$ and $2 s 3 d$ configurations. For the former the singlet density is clearly higher than the triplet one. For the $2 s 3 d$ configuration the triplet shows slightly higher values than the singlet state. For the other configurations the statistical errors associated to the Monte Carlo calculation do no allow us to appreciate clearly the differences between singlet and triplet single-particle density for short distances. The configurations with an electron in the $\mathrm{M}$ shell behave differently than the $2 s 2 p$ and $2 p^{2}$ ones at low and medium distances, where the difference function show a region where it is positive. That means that for those configurations the singlet states have a higher density at both low and large electron-nucleus distances, whereas for the $2 s 2 p$ and $2 p^{2}$ the singlet density is clearly smaller than the triplet one at low and medium $r$-distances. It is evident from this figure that the decrease in the value $\left\langle r^{-1}\right\rangle$ for singlet states is a much more complex electronic redistribution than the one observed for the $2 s 2 p$ [12, 14].

At the middle of Figure 3 we plot the difference function for the intracule density. Again the behavior of the $2 s 2 p$ and $2 p^{2}$ difference functions is very different to the rest of the cases studied. The first two configurations show a deep minimum at short interelectronic distances that indicates that, despite the Fermi hole, electrons are more likely to be at short interelectronic distances in the triplet state than in the singlet one. However the situation is different in the other configurations where the electronic radial intracule density is higher in singlet states for short and large interelectronic distances.

At this point it is important to note the difference in magnitude between the $2 s 2 p$ and $2 p^{2}$ configurations. The first one shows a relatively deeper hole for the single-particle than for the intracule density that allows one to explain the Hund's rule as usual. However for the $2 p^{2}$ configuration the magnitude of the hole in the intracule density is much greater than the corresponding one in the single particle density. This means that the electron-electron repulsion energy is much smaller in the singlet than in the triplet state, leading to an inversion in the order of the states.

The extracule density give us further insight into these facts. For the $2 s 2 p$ configuration, the electrons in the triplet have a tendency to be at opposite positions with respect to the nucleus to reduce the increased electron repulsion energy [14]. This effect is much less pronounced in the $2 p^{2}$ configuration as can be seen in Figure 3 (bottom), while for the rest of the configurations the opposite holds.

The results obtained for the $2 s 2 p$ configuration show the same behavior as in the HartreeFock framework [14], although the numerical values are different. These two two-body 
difference functions decrease from zero to a minimum value, then increase to a maximum from where they tends to zero taking positive values. The three densities of the $2 s 3 p$ configuration show a minimum at short distances, not very important in magnitude. This is due to the different behavior of singlet and triplet densities at their corresponding second maximum, making this configuration slightly different from all the rest.

Finally, in all cases the difference functions approach to zero at large distances from positive values, that indicates that singlet states are more extended in space than triplet ones.

To better understand these differences, we show in Figures 4, 5 and 6, the singlet and triplet single-particle, intracule and extracule radial densities, respectively for each one of the configurations studied here. In all cases the pictures show two or three maxima, corresponding to the number of shells involved in each configuration. The third maximum is more difficult to observe in the single-particle density, as it can be seen in Figure 3, but it is very important in both the intracule and extracule densities. In all the cases considered the more important differences are found for the second and third maxima due to the different coupling of the outermost electrons. For the $2 s 2 p$ and $2 p^{2}$ configurations, the second maximum of the single particle density is higher for the triplet than for the singlet state. For the other configurations the second maximum is practically the same for the singlet and the triplet while the third maximum is again higher for the triplet than for the singlet. For both the intracule and the extracule densities the behavior is similar as in the single-particle density with some exceptions. For the $2 s 3 s$ and $2 s 3 d$ configurations in both the intracule and extracule densities the second maximum of the singlet is higher than its counterpart in the triplet state. For the $2 s 3 p$ states the second maximum of the extracule density is higher for the singlet than for the triplet, while the contrary holds for the intracule density. The differences for the single-particle density are less important than for the intracule or extracule densities.

In Table $\mathrm{V}$ we report some two body properties in position and momentum spaces. In particular we give the position expectation value $\left\langle\vec{r}_{1} \cdot \vec{r}_{2}\right\rangle=\left\langle\sum_{i>j} \vec{r}_{i} \cdot \vec{r}_{j}\right\rangle$ and the angular correlation factor, $\tau$, introduced by Kutzelnigg, Del Re, and Berthier [33, defined as

$$
\tau=\frac{2 \sum_{i>j}\left\langle\vec{r}_{i} \cdot \vec{r}_{j}\right\rangle}{(N-1) \sum_{i}\left\langle r_{i}^{2}\right\rangle}
$$

This quantity is bounded in magnitude by unity, $-1 \leq \tau \leq 1 . \tau=1(\tau=-1)$ means perfect positive (negative) correlation and $\tau=0$ is for non-correlated variables. For atomic systems this coefficient is a measure of the averaged relative angle of the position vectors of each pair of electrons, and it is related to quantities that may be obtained from experimental measurements, such as the diamagnetic susceptibility and the dipole oscillator strength distribution. Similar definitions are done in momentum space. 
In position space these results indicate that the states here considered present negative angular correlation, except for the singlet from $2 s 2 p$ and $2 s 3 d$. This is consistent with the previous analysis carried out for the extracule density. In momentum space all the states show positive angular correlation. We have checked that these values depend strongly of the configuration mixing chosen in such a way that a small difference in energy can lead to a difference of about $5 \%$ for these coefficients.

We also report in Table $\mathrm{V}$ some correlated momentum expectation values which are directly obtained in the Monte Carlo calculation. In particular we show the expectation values $\left\langle p^{2}\right\rangle$, $\left\langle p_{12}^{2}\right\rangle,\left\langle P^{2}\right\rangle$ and $\left\langle\vec{p}_{1} \cdot \vec{p}_{2}\right\rangle$ These values are compared with those obtained from $\Phi_{m c}$, i.e. with those obtained from a multiconfiguration non explicitly correlated wave function. As it is known these two body expectation values as well as $\left\langle p^{2}\right\rangle$ are not linearly independent, but they satisfy some relations that, for four electron atoms, are written as [36]

$$
\begin{gathered}
3\left\langle p^{2}\right\rangle=\left\langle p_{12}^{2}\right\rangle+2\left\langle\vec{p}_{1} \cdot \vec{p}_{2}\right\rangle \\
3\left\langle p^{2}\right\rangle=4\left\langle P^{2}\right\rangle-2\left\langle\vec{p}_{1} \cdot \vec{p}_{2}\right\rangle
\end{gathered}
$$

As can be noticed from Table $\mathrm{V}$ the effects of electronic correlations are very important for $\left\langle p^{2}\right\rangle$ and henceforth for the kinetic energy. This can be analyzed in terms of the previous equations. The value of $\left\langle\vec{p}_{1} \cdot \vec{p}_{2}\right\rangle$ from a non correlated wave function is nearly zero, except for the $2 s 2 p-{ }^{3} \mathrm{P}$ state and for the $2 p^{2}$ terms, and becomes positive when correlations are taken into account. Electronic correlations also increase $\left\langle P^{2}\right\rangle$ while reduce the value of $\left\langle p_{12}^{2}\right\rangle$. This same trend was also found for the ground state of the isoelectronic series of the beryllium atom [36]. Finally it is worth to stress that the inclusion of the Jastrow correlation factor reveals to be very important in order to get a reliable description of these properties.

\section{CONCLUSIONS}

The first excited states of the beryllium atom have been studied. We have considered the singlet and triplet states of the configurations $2 s 2 p\left({ }^{3} P\right.$ and $\left.{ }^{1} P\right), 2 s 3 s\left({ }^{3} S\right.$ and $\left.{ }^{1} S\right), 2 p^{2}$ $\left({ }^{1} D\right.$ and $\left.{ }^{3} P\right) 2 s 3 p\left({ }^{3} P\right.$ and $\left.{ }^{1} P\right)$ and $2 s 3 d\left({ }^{3} D\right.$ and $\left.{ }^{1} D\right)$. We have analyzed the differences between singlet and triplet states and the effect of electronic correlations on them. This study has been carried out not only for the energy of the state but also on some other properties related to the electronic distribution. Explicitly correlated wave functions have been used to describe these states. They include a Jastrow-type correlation factor and a multiconfiguration model wave function providing accurate values of the correlation energy. The model wave function has been calculated by a generalization of the optimized effective potential method to include configuration interaction type expansions. All the calculations of this work have been performed by using the Variational Monte Carlo Method.

The effects of electronic correlations are more important for singlet than for triplet states. 
To describe the former it is absolutely necessary to consider a multi configuration model wave function for any of the states studied, while for the latter a single configuration model wave function provides practically the same results as those given by the multi configuration one. Both the correlation factor, $F$, and the Coulomb hole, show a similar structure for singlet and triplet states. The effects of electronic correlations are more important for the intracule and extracule densities than for the single-particle density, as one could expect.

Singlet states present a more diffuse electronic distribution than triplet ones. This effect is overestimated in the Hartree-Fock approximation. This fact is deeply related to the relative binding energy of the singlet and triplet states because of its influence on the balance between the nuclear attraction and electron-electron repulsion energies. For the $2 s 2 p$ and $2 s 3 s$ configurations we have found that the bigger magnitude of the nuclear attraction in the triplet compensates the bigger value of the electronic repulsion of the triplet giving rise to a lower value of the binding energy, according to the usual interpretation of Hund's rule. This is not the case of the $2 s 3 p$ configuration where the lower energy of the triplet state is due to the bigger electronic repulsion in the singlet, where the nuclear attraction is also slightly bigger in magnitude than in the triplet. For the $2 s 3 d$ configuration both the electron-nucleus attraction and the electron-electron repulsion contribute in the same direction to make more bound the triplet state. The $2 p^{2}$ configuration does not satisfy the Hund's rule because here the bigger magnitude of the nuclear attraction in the triplet does not compensate its bigger value of the electronic repulsion.

The effect of electronic correlations is also to decrease the expectation value $\left\langle\vec{r}_{1} \cdot \vec{r}_{2}\right\rangle$, i.e. to increase the probability of two electrons to be at opposite positions with respect to the nucleus, except for the singlet $2 s 2 p$ and $2 s 3 d$. In momentum space the situation is the opposite, finding positive angular correlation. This is related to the increase of the kinetic energy when correlations are taken into account (virial theorem) and the pair-center of mass momentum distribution, while the relative momentum of two electrons becomes reduced.

\section{ACKNOWLEDGMENTS}

This work has been partially supported by the Spanish Dirección General de Investigación Científica y Técnica (DGICYT) under contract PB98-1318 and by the Junta de Andalucía. A.S. acknowledges the Italian MURST for financial support from the grant MIUR-2001025498. 


\section{References}

[1] R. L. Graham, D. L. Yeager, J. Olsen, P. Jorgensen, R. Harrison, S. Zarrabian and R. Bartlett, J. Chem. Phys. 85, 6544 (1986)

[2] L. Bertini, M. Mella, D. Bressanini and G. Morosi, J. Phys. B, 34257 (2001).

[3] J. Komasa, J. Rychlewsky and K. Jankowski, Phys. Rev. A, 65042507 (2002).

[4] C. A. Coulson and A. H. Neilson, Proc. Phys. Soc. 78, 831 (1961)

[5] R. J. Boyd and C. A. Coulson, J. Phys. B, 6, 782 (1973)

[6] R. J. Boyd, Nature, 310, 480 (1984)

[7] F. Arias de Saavedra, E. Buendía, F. J. Gálvez and I. Porras, J. Phys. B 29, 3803 (1996).

[8] P. E. Regier and A. J. Thakkar, J. Phys. B, 17, 3391 (1984), and references therein.

[9] D. R. Hartree and W. Hartree, Proc. Roy. Soc. (Lond.) A 154, 588 (1936)

[10] Ch. Froese, J. Chem. Phys. 47, 4010 (1967)

[11] H. Tatewaki, H. Taketa and F. Sasaki, Int. J. Quantum Chem. 5, 335 (1971)

[12] H. Tatewaki and K. Tanaka, J. Chem. Phys. 60, 601 (1974)

[13] I. Shim and J. P. Dahl, Theor. Chem. Acta 48, 165 (1978)

[14] T. Koga, H. Matsuyama, J. Molina and J. S. Dehesa, Eur. Phys. J. D 7, 17 (1999)

[15] T. Koga, H. Matsuyama, J. S. Dehesa and A. J. Thakkar, J. Chem. Phys. 110, 5763 (1999)

[16] K. V. Darvesh and R. J. Boyd, J. Chem. Phys. 87, 5329 (1987), and references therein.

[17] F. J. Gálvez, E. Buendía and A. Sarsa J. Chem. Phys. 111, 10903 (1999).

[18] B. L. Hammond, W. A. Lester Jr and P. J. Reynolds, Monte Carlo methods in ab initio quantum chemistry, World Scientific, Singapore (1994).

[19] S. F. Boys and N. C. Handy, Proc. R. Soc. London Ser. A 310, 43 (1969).

[20] K. E. Schmidt and J. W. Moskowitz, J. Chem. Phys., 93, 4172 (1990).

[21] J. C. Slater, Phys. Rev. 81, 385 (1951). 
[22] J. D. Talman and W. F. Shadwick, Phys. Rev. A 14, 36 (1976)

[23] K. Aashanar, T. M. Luke and J. D. Talman, At. Data Nucl. Data Tables 22, 443 (1978)

[24] R. Colle and R. K. Nesbet, J. Phys. B 34, 2475 (2001).

[25] E. R. Davidson, S. A. Hasgtrom, S. J. Chakravorty, V. M. Umar and C. F. Fisher, Phys. Rev A 44, 7071 (1991)

[26] F. J. Gálvez, E. Buendía and A. Sarsa, J. Chem. Phys. 115, 1166 (2001).

[27] X. Lin, H. Zhang and A. M. Rappe, J. Chem. Phys., 112, 2650 (2000).

[28] S. Bashkin and J. A. Stoner, Jr., Atomic Energy Levels and Grotriam Diagrams I (North Holland, Amsterdam, 1975).

[29] P. Langfelder, S. M. Rothstein and J. Vrbik, J. Chem. Phys. 107, 8525 (1997).

[30] A. Sarsa, F. J. Gálvez and E. Buendía, J. Chem. Phys. 109, 7075 (1998).

[31] J. Komasa, W. Cencek and J. Rychlewski, Phys. Rev A93, 4500 (1995).

[32] T. Koga, J. Chem. Phys. 116, 6614 (2002)

[33] W. Kutzelnigg, G. Del Re and G. Berthier, Phys. Rev. 172, 49 (1969).

[34] A. J. Thakkar, Phys. Rev. A23, 473 (1981).

[35] A. J. Thakkar, Phys. Rev. A25, 1820 (1982).

[36] F. J. Gálvez, E. Buendía and A. Sarsa J. Chem. Phys. 113, 8631 (2000). 


\section{TABLE CAPTIONS}

Table I: Excitation energy (in eV) of the different states of beryllium obtained from $\Phi_{m c}$ $\left(E_{m c}\right)$ and $\Psi_{c m c}\left(E_{c m c}\right)$ as compared with those of [28] $\left(E_{\text {exact }}\right)$. In parentheses we give the statistical error in the last digit, and in bracket is shown the percentage of correlation energy recovered in each calculation. The configurations used in the model wave function are also shown with the core electrons $1 s^{2}$ implicit in the notation.

Table II: Radial moments and value at the origin of the single-particle density for the different states studied in this work. For the ground state the results are compared with those of [31], which can be considered as exact, and with the VMC of [26]. For the $2 \mathrm{~s} 2 \mathrm{p}$ states some HF results are also shown. In parentheses we give the statistical error in the last digit.

Table III: Radial moments and value at the origin of the intracule density. For the ground state the results are compared with those of [31], which can be considered as exact, and with those of [26]. For the 2s2p states some HF and CI results are also shown. In parentheses we give the statistical error in the last digit.

Table IV: Radial moments and value at the origin of the extracule density. For the $2 \mathrm{~s} 2 \mathrm{p}$ states some HF results are also shown. In parentheses we give the statistical error in the last digit.

Table V: Several two body position and momentum properties obtained from $\Psi_{c m c}$ as compared with those obtained from $\Phi_{m c}$ that does not include the Jastrow factor. In parentheses we give the statistical error in the last digit. 


\section{FIGURE CAPTIONS}

FIGURE 1: Difference functions between the fully-correlated and the self consistent results (cmc-sc) and between the multiconfiguration and self consistent ones (mc-sc) for the radial single-particle (top), intracule (middle) and extracule (bottom) densities. The results for the $2 \mathrm{~s} 2 \mathrm{p}-{ }^{1} \mathrm{P}\left(2 \mathrm{~s} 2 \mathrm{p}-{ }^{3} \mathrm{P}\right)$ are shown in the left (right) part of the Figure.

FIGURE 2: Difference functions $f_{c m c-m c}(t)$ between the fully correlated and the multiconfiguration radial single-particle (top), intracule (middle) and extracule (bottom) densities for the $2 s 2 p$ states.

FIGURE 3: Difference between singlet and triplet radial single-particle (top), intracule (middle) and extracule (bottom) densities.

Figure 4: Comparison between singlet and triplet radial single particle density for the different excited states calculated from the best trial wave function obtained in this work.

FIGURE 5: Comparison between singlet and triplet radial intracule density for the different excited states calculated from the best trial wave function obtained in this work.

FIGURE 6: Comparison between singlet and triplet radial extracule density for the different excited states calculated from the best trial wave function obtained in this work. 
TABLE I

\begin{tabular}{l|l|lll}
\hline \hline & Configurations & $E_{m c}$ & $E_{c m c}$ & $E_{\text {exact }}$ \\
\hline $2 \mathrm{~s}^{2}\left({ }^{1} \mathrm{~S}\right)$ & $2 \mathrm{~s}^{2} ; 2 \mathrm{p}^{2} ; 2 \mathrm{~s} 3 \mathrm{~s}$ & 1.383 & $0.069(1)[95.7]$ & 0 \\
\hline 2s2p $\left({ }^{3} \mathrm{P}\right)$ & $2 \mathrm{~s} 2 \mathrm{p} ; 2 \mathrm{~s} 3 \mathrm{p} ; 3 \mathrm{~s} 2 \mathrm{p} ; 2 \mathrm{p} 3 \mathrm{~d}$ & 4.147 & $2.795(1)[95.0]$ & 2.725 \\
\hline $2 \mathrm{~s} 2 \mathrm{p}\left({ }^{1} \mathrm{P}\right)$ & $2 \mathrm{~s} 2 \mathrm{p} ; 2 \mathrm{~s} 3 \mathrm{p} ; 2 \mathrm{p} 3 \mathrm{~d}$ & 6.845 & $5.405(1)[91.8]$ & 5.277 \\
\hline $2 \mathrm{~s} 3 \mathrm{~s}\left({ }^{3} \mathrm{~S}\right)$ & $2 \mathrm{~s} 3 \mathrm{~s} ; 2 \mathrm{p} 3 \mathrm{p}$ & 7.796 & $6.528(1)[94.7]$ & 6.457 \\
\hline $2 \mathrm{~s} 3 \mathrm{~s}\left({ }^{1} \mathrm{~S}\right)$ & $2 \mathrm{~s}^{2} ; 2 \mathrm{p}^{2} ; 2 \mathrm{~s} 3 \mathrm{~s} ; 2 \mathrm{p} 3 \mathrm{p}$ & 8.245 & $6.875(1)[93.4]$ & 6.779 \\
\hline $2 \mathrm{p}^{2}\left({ }^{1} \mathrm{D}\right)$ & $2 \mathrm{p}^{2} ; 2 \mathrm{~s} 3 \mathrm{~d} ; 2 \mathrm{~s} 4 \mathrm{~d} ; 2 \mathrm{p} 3 \mathrm{p}$ & 8.460 & $7.157(2)[92.4]$ & 7.05 \\
\hline $2 \mathrm{~s} 3 \mathrm{p}\left({ }^{3} \mathrm{P}\right)$ & $2 \mathrm{~s} 3 \mathrm{p} ; 2 \mathrm{~s} 2 \mathrm{p} ; 3 \mathrm{~s} 3 \mathrm{p}$ & 8.679 & $7.403(3)[92.7]$ & 7.303 \\
\hline $2 \mathrm{p}^{2}\left({ }^{3} \mathrm{P}\right)$ & $2 \mathrm{p}^{2} ; 3 \mathrm{p} 2 ; 3 \mathrm{~d}{ }^{2}$ & 8.823 & $7.457(2)[96.1]$ & 7.401 \\
\hline $2 \mathrm{~s} 3 \mathrm{p}\left({ }^{1} \mathrm{P}\right)$ & $2 \mathrm{~s} 3 \mathrm{p} ; 2 \mathrm{~s} 2 \mathrm{p} ; 3 \mathrm{~s} 2 \mathrm{p} ; 3 \mathrm{~s} 3 \mathrm{p} ; 2 \mathrm{p} 3 \mathrm{~d}$ & 8.926 & $7.604(1)[90.3]$ & 7.462 \\
\hline $2 \mathrm{~s} 3 \mathrm{~d}\left({ }^{3} \mathrm{D}\right)$ & $2 \mathrm{~s} 3 \mathrm{~d} ; 3 \mathrm{~s} 2 \mathrm{~d} ; 2 \mathrm{p} 3 \mathrm{p}$ & 9.055 & $7.760(1)[95.1]$ & 7.694 \\
\hline $2 \mathrm{~s} 3 \mathrm{~d}\left({ }^{1} \mathrm{D}\right)$ & $2 \mathrm{~s} 3 \mathrm{~d} ; 2 \mathrm{p}^{2} ; 2 \mathrm{p} 3 \mathrm{p} ; 2 \mathrm{~s} 4 \mathrm{~d}$ & 9.373 & $8.116(2)[91.5]$ & 7.998 \\
\hline \hline
\end{tabular}


TABLE II

\begin{tabular}{|c|c|c|c|c|c|c|}
\hline & $\overline{c \rho(0)}$ & $\left\langle r^{-2}\right\rangle$ & $\left\langle r^{-1}\right\rangle$ & $\overline{\langle r\rangle}$ & $\left\langle r^{2}\right\rangle$ & $\overline{\left\langle r^{3}\right\rangle}$ \\
\hline $2 \mathrm{~s}^{2}\left({ }^{1} \mathrm{~S}\right)$ & $35.2(1)$ & $57.3(2)$ & $8.427(1)$ & $5.9792(5)$ & $16.254(3)$ & $56.55(2)$ \\
\hline Exact 31 & 35.3116 & 57.59808 & 8.42735 & 5.97256 & 16.2476 & 56.772 \\
\hline 26 & $35.3(1)$ & $57.4(2)$ & $8.433(2)$ & $5.985(1)$ & $16.343(9)$ & $57.43(6)$ \\
\hline $2 \mathrm{~s} 2 \mathrm{p}\left({ }^{3} \mathrm{P}\right)$ & $34.7(1)$ & $56.8(2)$ & $8.3601(8)$ & $6.2160(6)$ & $18.111(4)$ & $68.81(3)$ \\
\hline HF 14 & & & & 5.41967 & 17.88637 & \\
\hline $2 \mathrm{~s} 2 \mathrm{p}\left({ }^{1} \mathrm{P}\right)$ & $34.7(1)$ & $56.6(2)$ & $8.273(1)$ & $7.240(1)$ & $27.83(1)$ & $152.0(2)$ \\
\hline HF 14 & & & & 7.33567 & 37.49063 & \\
\hline $2 \mathrm{~s} 3 \mathrm{~s}\left({ }^{3} \mathrm{~S}\right)$ & $34.94(9)$ & $56.6(1)$ & $8.144(1)$ & $10.818(2)$ & $75.03(4)$ & $705.2(8)$ \\
\hline $2 \mathrm{~s} 3 \mathrm{~s}\left({ }^{1} \mathrm{~S}\right)$ & $35.3(2)$ & $57.3(2)$ & $8.1328(9)$ & $11.804(5)$ & $95.85(8)$ & $1067(1)$ \\
\hline $2 \mathrm{p}^{2}\left({ }^{1} \mathrm{D}\right)$ & $34.8(3)$ & $56.5(4)$ & $8.202(1)$ & $7.983(2)$ & $36.71(3)$ & $248.5(4)$ \\
\hline $2 \mathrm{~s} 3 \mathrm{p}\left({ }^{3} \mathrm{P}\right)$ & $34.4(1)$ & $56.1(1)$ & $8.108(1)$ & $13.017(4)$ & $120.66(9)$ & $1498(2)$ \\
\hline $2 \mathrm{p}^{2}\left({ }^{3} \mathrm{P}\right)$ & $34.0(2)$ & $56.1(3)$ & $8.250(1)$ & $6.7163(7)$ & $22.080(6)$ & $95.81(5)$ \\
\hline $2 \mathrm{~s} 3 \mathrm{p}\left({ }^{1} \mathrm{P}\right)$ & $34.9(1)$ & $56.6(1)$ & $8.111(1)$ & $13.686(5)$ & $142.5(1)$ & $2020(3)$ \\
\hline $2 \mathrm{~s} 3 \mathrm{~d}\left({ }^{3} \mathrm{D}\right)$ & $35.0(1)$ & $56.9(2)$ & $8.093(1)$ & $12.805(2)$ & $114.27(2)$ & $1368(2)$ \\
\hline $2 \mathrm{~s} 3 \mathrm{~d}\left({ }^{1} \mathrm{D}\right)$ & $34.7(2)$ & $56.7(3)$ & $8.089(1)$ & $15.457(7)$ & $193.7(2)$ & $3254(5)$ \\
\hline
\end{tabular}


TABLE III

\begin{tabular}{|c|c|c|c|c|c|c|}
\hline & $\overline{h(0)}$ & $\overline{\left\langle\left\langle u^{-2}\right\rangle\right.}$ & $\left\langle u^{-1}\right\rangle$ & $\langle u\rangle$ & $\left\langle\overline{\left\langle u^{2}\right\rangle}\right.$ & $\left\langle\overline{\left\langle u^{3}\right\rangle}\right.$ \\
\hline $2 \mathrm{~s}^{2}\left({ }^{1} \mathrm{~S}\right)$ & $1.6079(8)$ & $9.542(9)$ & $4.3741(4)$ & $15.292(1)$ & $52.97(1)$ & $223.07(8)$ \\
\hline Exact 31] & 60704 & 67 & 4.3747 & 15.272 & 52.85 & 222.486 \\
\hline & $1.611(1)$ & $9.55(1)$ & $4.375(1)$ & $15.305(3)$ & $53.15(3)$ & $224.7(2)$ \\
\hline MCHF [32] & & 9.64232 & 4.38013 & 15.2804 & 52.9295 & 223.015 \\
\hline $2 \mathrm{~s} 2 \mathrm{p}\left({ }^{3} \mathrm{P}\right)$ & $1.571(1)$ & $9.37(1)$ & $4.2974(3)$ & $16.116(2)$ & $60.17(2)$ & $276.7(1)$ \\
\hline CI 12 & & & 4.374 & 16.206 & & \\
\hline $\mathrm{HF}$ [14] & & 10.31304 & 4.388274 & 16.04838 & 59.72532 & \\
\hline $2 \mathrm{~s} 2 \mathrm{p}\left({ }^{1} \mathrm{P}\right)$ & $1.5757(8)$ & $9.24(1)$ & $4.1434(3)$ & $18.261(4)$ & $82.96(5)$ & $493.4(6)$ \\
\hline CI |12 & & & 4.206 & 18.89 & & \\
\hline $\mathrm{HF}$ [14] & & 10.15692 & 4.122636 & 20.64546 & 111.0888 & \\
\hline $2 \mathrm{~s} 3 \mathrm{~s}\left({ }^{3} \mathrm{~S}\right)$ & $1.589(3)$ & $9.05(3)$ & $3.7094(3)$ & $29.127(6)$ & $230.3(1)$ & $2264(2)$ \\
\hline $2 \mathrm{~s} 3 \mathrm{~s}\left({ }^{1} \mathrm{~S}\right)$ & $1.589(4)$ & $9.02(2)$ & $3.6874(7)$ & $31.96(2)$ & $292.2(3)$ & $3365(5)$ \\
\hline $2 \mathrm{p}^{2}\left({ }^{1} \mathrm{D}\right)$ & $1.548(5)$ & $9.16(6)$ & $3.9798(3)$ & $21.212(6)$ & 118.4(1) & $875(1)$ \\
\hline $2 \mathrm{~s} 3 \mathrm{p}\left({ }^{3} \mathrm{P}\right)$ & $1.592(1)$ & $9.00(1)$ & $3.6367(4)$ & $35.38(1)$ & $363.3(3)$ & $4627(6)$ \\
\hline $2 \mathrm{p}^{2}\left({ }^{3} \mathrm{P}\right)$ & $1.518(1)$ & $9.16(2)$ & $4.1997(3)$ & $17.01(2)$ & $67.56(2)$ & $329.6(2)$ \\
\hline $2 \mathrm{~s} 3 \mathrm{p}\left({ }^{1} \mathrm{P}\right)$ & $1.588(1)$ & $9.03(2)$ & $3.6536(4)$ & $37.47(1)$ & $430.6(3)$ & $6230(9)$ \\
\hline $2 \mathrm{~s} 3 \mathrm{~d}\left({ }^{3} \mathrm{D}\right)$ & $1.586(1)$ & $8.93(1)$ & $3.6011(2)$ & $34.804(7)$ & $345.7(2)$ & $4259(4)$ \\
\hline $2 \mathrm{~s} 3 \mathrm{~d}\left({ }^{1} \mathrm{D}\right)$ & $1.584(1)$ & $8.99(2)$ & $3.6058(4)$ & $42.58(2)$ & $580.9(6)$ & 9908(16) \\
\hline
\end{tabular}


TABLE IV

\begin{tabular}{l|llllll}
\hline \hline & $d(0)$ & $\left\langle R^{-2}\right\rangle$ & $\left\langle R^{-1}\right\rangle$ & $\langle R\rangle$ & $\left\langle R^{2}\right\rangle$ & $\left\langle R^{3}\right\rangle$ \\
\hline $2 \mathrm{~s}^{2}\left({ }^{1} \mathrm{~S}\right)$ & $16.873(5)$ & $43.17(9)$ & $9.2717(7)$ & $7.0712(6)$ & $11.139(2)$ & $21.077(8)$ \\
$\mathrm{MCHF}$ [32] & & 42.9430 & 9.26995 & 7.07675 & 11.1826 & 21.2990 \\
\hline 2s2p $\left({ }^{3} \mathrm{P}\right)$ & $16.564(4)$ & $42.56(6)$ & $9.1955(6)$ & $7.3087(7)$ & $12.124(3)$ & $24.48(1)$ \\
$\mathrm{HF}[14]$ & & 42.29016 & 9.09576 & 7.43478 & 12.59922 & \\
\hline 2s2p ( $\left.{ }^{1} \mathrm{P}\right)$ & $16.461(4)$ & $40.97(7)$ & $8.4700(6)$ & $9.201(2)$ & $21.00(1)$ & $62.18(6)$ \\
$\mathrm{HF}[14]$ & & 40.398 & 8.12412 & 10.64142 & 29.16552 & \\
\hline 2s3s $\left({ }^{3} \mathrm{~S}\right)$ & $16.555(4)$ & $40.11(7)$ & $7.6748(5)$ & $14.201(3)$ & $54.98(3)$ & $266.8(3)$ \\
\hline 2s3s $\left({ }^{1} \mathrm{~S}\right)$ & $16.545(5)$ & $40.24(7)$ & $7.657(2)$ & $15.653(7)$ & $70.72(6)$ & $405.1(6)$ \\
\hline 2p ${ }^{2}\left({ }^{1} \mathrm{D}\right)$ & $16.351(5)$ & $41.1(1)$ & $8.4431(8)$ & $9.796(3)$ & $25.46(2)$ & $90.3(1)$ \\
\hline 2s3p $\left({ }^{3} \mathrm{P}\right)$ & $16.558(5)$ & $40.14(8)$ & $7.5191(8)$ & $17.585(5)$ & $90.16(6)$ & $573.6(7)$ \\
\hline 2p ${ }^{2}\left({ }^{3} \mathrm{P}\right)$ & $16.042(6)$ & $41.3(1)$ & $8.7389(5)$ & $8.334(1)$ & $16.230(5)$ & $38.75(2)$ \\
\hline 2s3p $\left({ }^{1} \mathrm{P}\right)$ & $16.538(5)$ & $40.3(1)$ & $7.5318(8)$ & $18.556(7)$ & $106.15(9)$ & $767(1)$ \\
\hline 2s3d $\left({ }^{3} \mathrm{D}\right)$ & $16.475(4)$ & $39.68(7)$ & $7.4332(6)$ & $17.211(4)$ & $84.96(5)$ & $521.9(5)$ \\
\hline 2s3d $\left({ }^{1} \mathrm{D}\right)$ & $16.526(4)$ & $40.09(9)$ & $7.4552(9)$ & $21.28(1)$ & $145.3(1)$ & $1239(2)$ \\
\hline \hline
\end{tabular}


TABLE V

\begin{tabular}{|c|c|c|c|c|c|c|c|}
\hline & $\left\langle\overline{\left.\vec{r}_{1} \cdot \overrightarrow{r_{2}}\right\rangle}\right.$ & 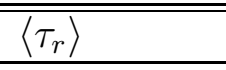 & 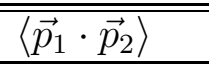 & 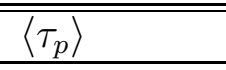 & 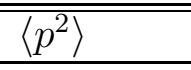 & 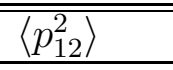 & $\overline{\left\langle\overline{\left\langle P^{2}\right\rangle}\right.}$ \\
\hline $2 \mathrm{~s}^{2}\left({ }^{1} \mathrm{~S}\right)$ & & & & & & & \\
\hline$\Psi_{c m c}$ & $-2.103(1)$ & $-0.08625(4)$ & $0.456(1)$ & $0.01036(3)$ & $29.342(8)$ & $87.11(2)$ & $22.234(6)$ \\
\hline$\Phi_{m c}$ & $-1.714(1)$ & $-0.07004(4)$ & $0.015(1)$ & $0.00034(3)$ & $29.301(7)$ & $87.87(2)$ & $21.983(5)$ \\
\hline $2 \mathrm{~s} 2 \mathrm{p}\left({ }^{3} \mathrm{P}\right)$ & & & & & & & \\
\hline$\Psi_{c m c}$ & $-2.920(1)$ & $-0.10747(4)$ & $0.260(1)$ & $0.00594(3)$ & $29.157(6)$ & $86.95(2)$ & $21.998(5)$ \\
\hline$\Phi_{m c}$ & $-3.051(1)$ & $-0.11350(4)$ & $-0.181(1)$ & $-0.00415(2)$ & $29.058(8)$ & $87.54(2)$ & $21.703(6)$ \\
\hline $2 \mathrm{~s} 2 \mathrm{p}\left({ }^{1} \mathrm{P}\right)$ & & & & & & & \\
\hline$\Psi_{c m c}$ & $0.258(2)$ & $0.00617(4)$ & $0.459(1)$ & $0.01057(2)$ & $28.962(7)$ & $85.97(2)$ & $21.951(5)$ \\
\hline$\Phi_{m c}$ & $-0.132(2)$ & $-0.00306(4)$ & $0.031(1)$ & $0.00071(2)$ & $28.799(7)$ & $86.33(2)$ & $21.615(5)$ \\
\hline $2 \mathrm{~s} 3 \mathrm{~s}\left({ }^{3} \mathrm{~S}\right)$ & & & & & & & \\
\hline$\Psi_{c m c}$ & $-2.578(3)$ & $-0.02291(3)$ & $0.449(1)$ & $0.01036(3)$ & $28.879(8)$ & $85.74(2)$ & $21.884(6)$ \\
\hline$\Phi_{m c}$ & $-2.690(3)$ & $-0.02430(3)$ & $0.013(1)$ & $0.00029(3)$ & $28.829(9)$ & $86.46(3)$ & $21.628(7)$ \\
\hline $2 \mathrm{~s} 3 \mathrm{~s}\left({ }^{1} \mathrm{~S}\right)$ & & & & & & & \\
\hline$\Psi_{c m c}$ & $-2.331(4)$ & $-0.01622(3)$ & $0.440(1)$ & $0.01016(3)$ & $28.858(7)$ & $85.69(2)$ & $21.864(5)$ \\
\hline$\Phi_{m c}$ & $0.048(5)$ & $0.00032(3)$ & $0.006(1)$ & $0.00015(3)$ & $28.731(8)$ & $86.18(2)$ & $21.551(6)$ \\
\hline $2 \mathrm{p}^{2}\left({ }^{1} \mathrm{D}\right)$ & & & & & & & \\
\hline$\Psi_{c m c}$ & $-4.140(2)$ & $-0.07518(6)$ & $0.151(1)$ & $0.00350(2)$ & $28.845(9)$ & $86.23(3)$ & $21.709(7)$ \\
\hline$\Phi_{m c}$ & $-4.042(2)$ & $-0.07229(6)$ & $-0.2978(9)$ & $-0.00691(2)$ & $28.738(8)$ & $86.81(2)$ & $21.405(6)$ \\
\hline $2 \mathrm{~s} 3 \mathrm{p}\left({ }^{3} \mathrm{P}\right)$ & & & & & & & \\
\hline$\Psi_{c m c}$ & $-0.673(4)$ & $-0.00372(2)$ & $0.425(1)$ & $0.00985(3)$ & $28.799(7)$ & $85.55(2)$ & $21.812(5)$ \\
\hline$\Phi_{m c}$ & $-0.026(5)$ & $-0.00014(3)$ & $-0.005(1)$ & $-0.00011(3)$ & $28.641(8)$ & $85.93(2)$ & $21.478(6)$ \\
\hline $2 \mathrm{p}^{2}\left({ }^{3} \mathrm{P}\right)$ & & & & & & & \\
\hline$\Psi_{c m c}$ & $-0.660(1)$ & $-0.01994(3)$ & $0.142(1)$ & $0.00329(3)$ & $28.815(7)$ & $86.16(2)$ & $21.682(6)$ \\
\hline$\Phi_{m c}$ & $-0.999(1)$ & $-0.03070(4)$ & $-0.282(1)$ & $-0.00658(3)$ & $28.573(8)$ & $86.28(2)$ & $21.289(6)$ \\
\hline $2 \mathrm{~s} 3 \mathrm{p}\left({ }^{1} \mathrm{P}\right)$ & & & & & & & \\
\hline$\Psi_{c m c}$ & $-1.512(6)$ & $-0.00707(3)$ & $0.409(1)$ & $0.00947(3)$ & $28.803(9)$ & $85.59(3)$ & $21.807(7)$ \\
\hline$\Phi_{m c}$ & $-1.212(5)$ & -0.005 & $-0.041(1)$ & $-0.00096(2)$ & $28.618(8)$ & $85.94(2)$ & $21.443(6)$ \\
\hline $2 \mathrm{~s} 3 \mathrm{~d}\left({ }^{3} \mathrm{D}\right)$ & & & & & & & \\
\hline$\Psi_{c m c}$ & $-1.474(5)$ & $-0.00860(3)$ & $0.440(1)$ & $0.01020(3)$ & $28.774(8)$ & $85.44(3)$ & $21.800(6)$ \\
\hline$\Phi_{m c}$ & $0.011(5)$ & $0.00006(3)$ & $0.005(1)$ & $0.00013(3)$ & $28.682(8)$ & $86.04(3)$ & $21.514(6)$ \\
\hline $2 \mathrm{~s} 3 \mathrm{~d}\left({ }^{1} \mathrm{D}\right)$ & & & & & & & \\
\hline$\Psi_{c m c}$ & $0.0953(6)$ & $0.00033(2)$ & $0.405(1)$ & $0.00939(3)$ & $28.766(7)$ & $85.49(2)$ & $21.777(6)$ \\
\hline$\Phi_{m c}$ & $0.367(6)$ & $0.00120(2)$ & $-0.050(1)$ & $-0.00115(3)$ & $28.771(8)$ & $86.41(2)$ & $21.554(6)$ \\
\hline
\end{tabular}



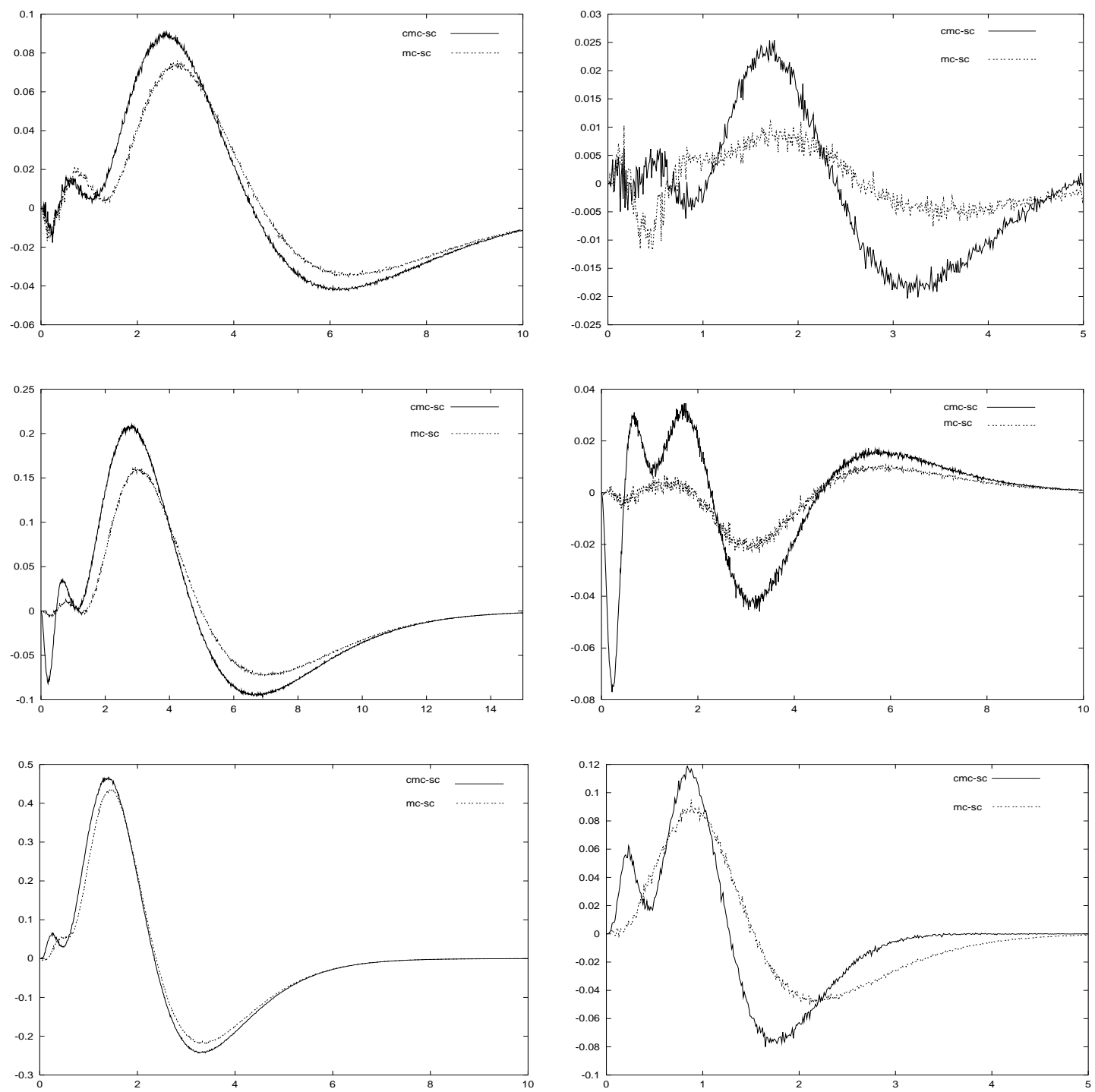

Figure 1 

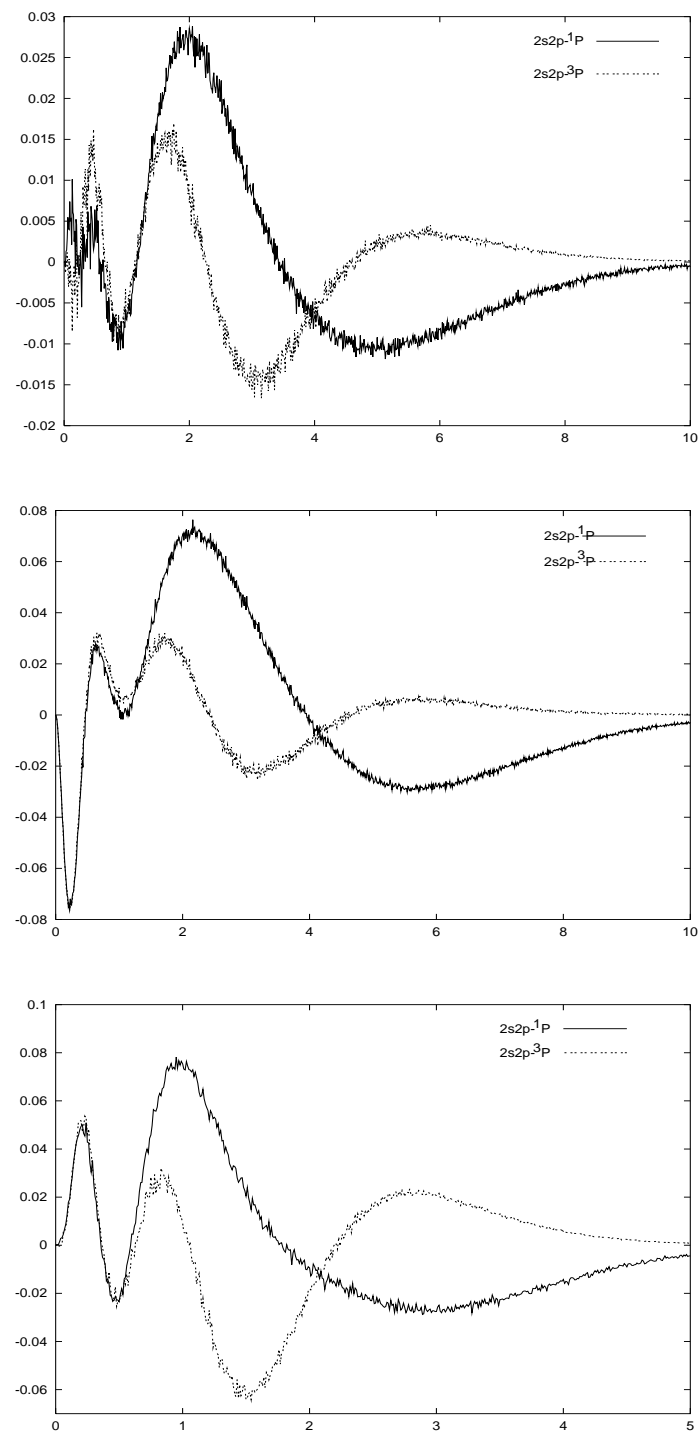

Figure 2 

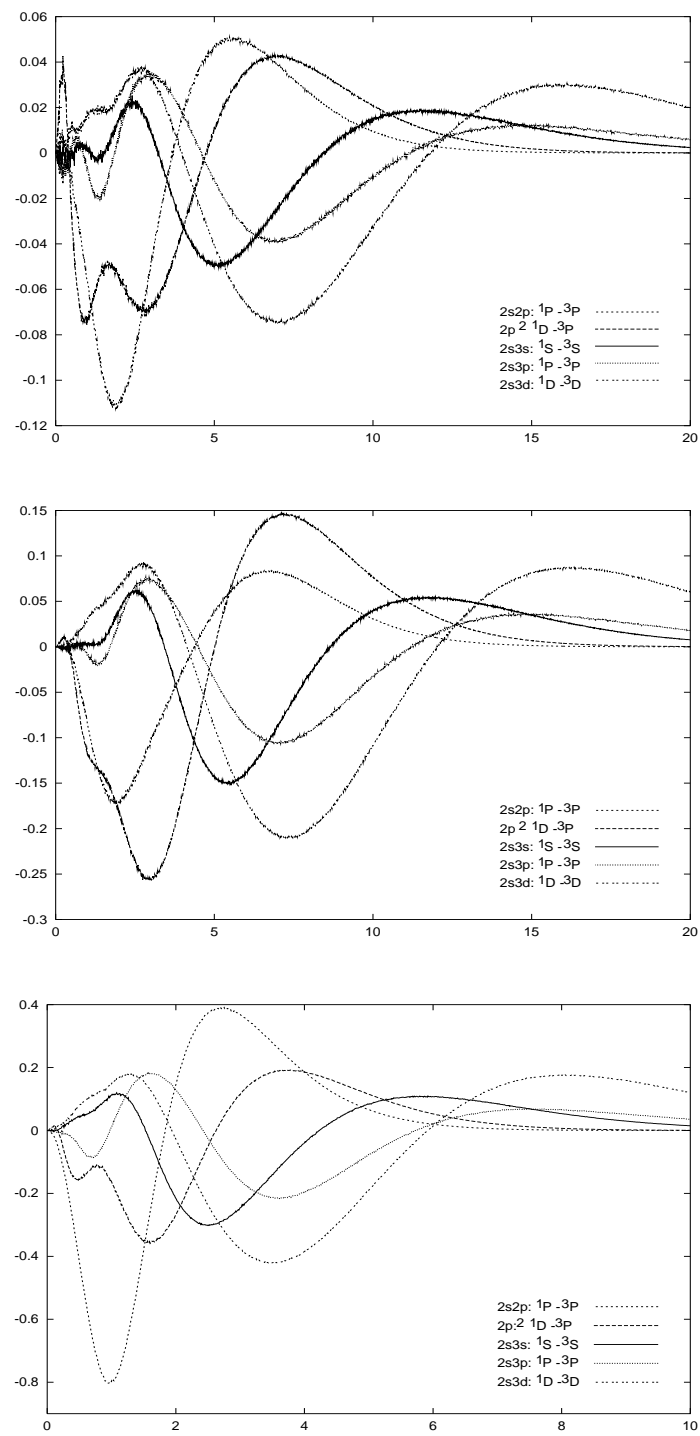

Figure 3 

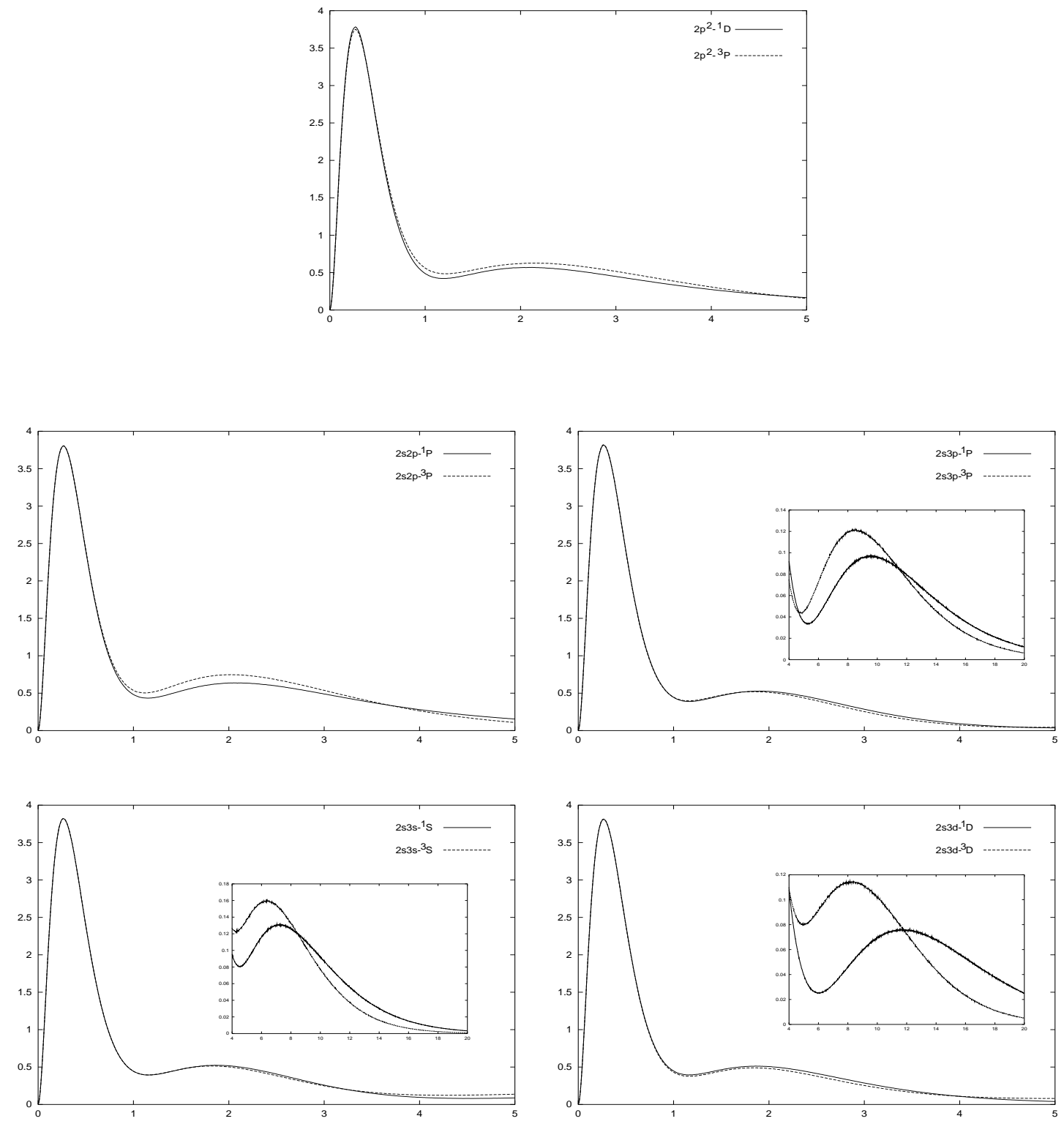

Figure 4 

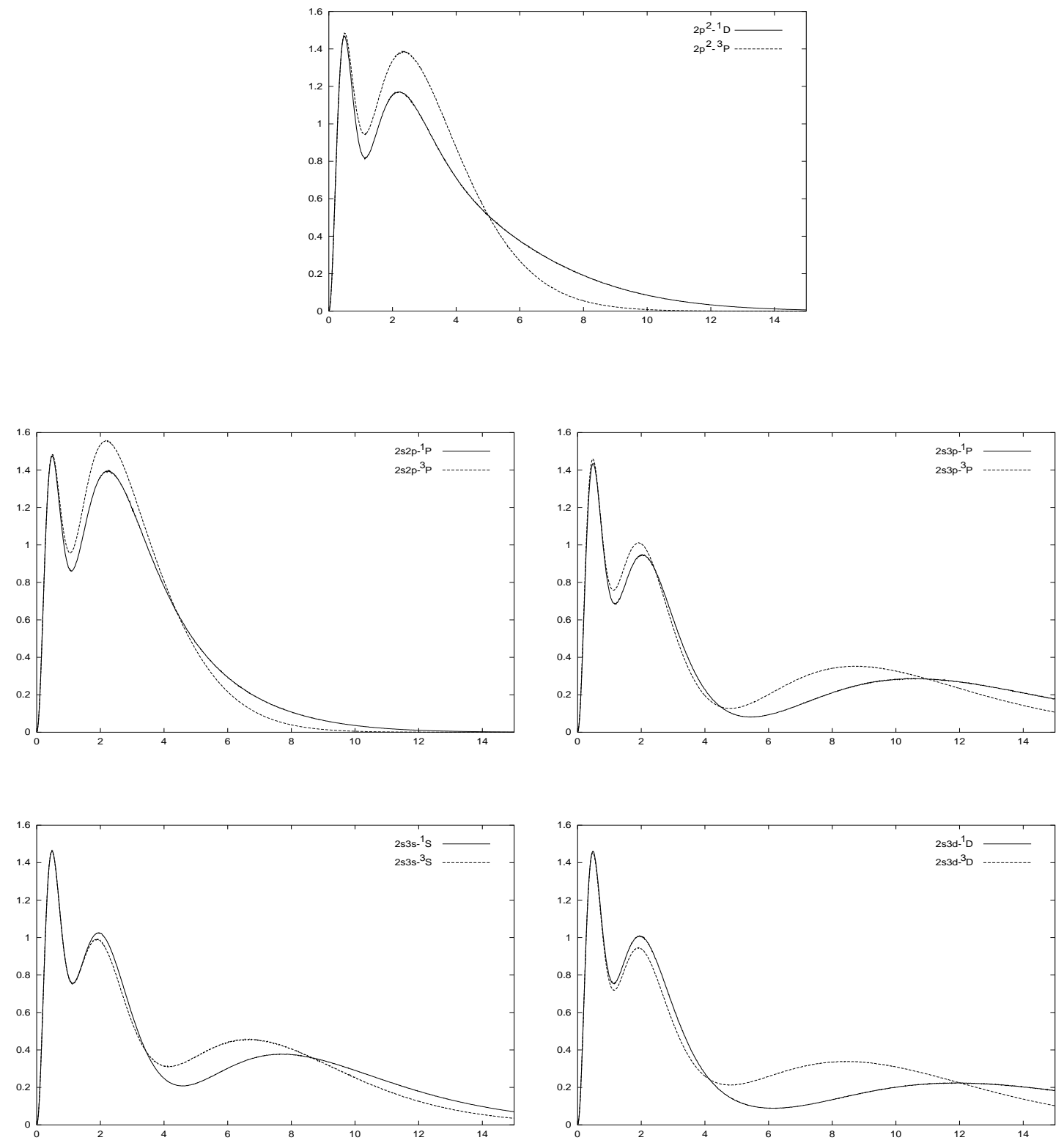

Figure 5 

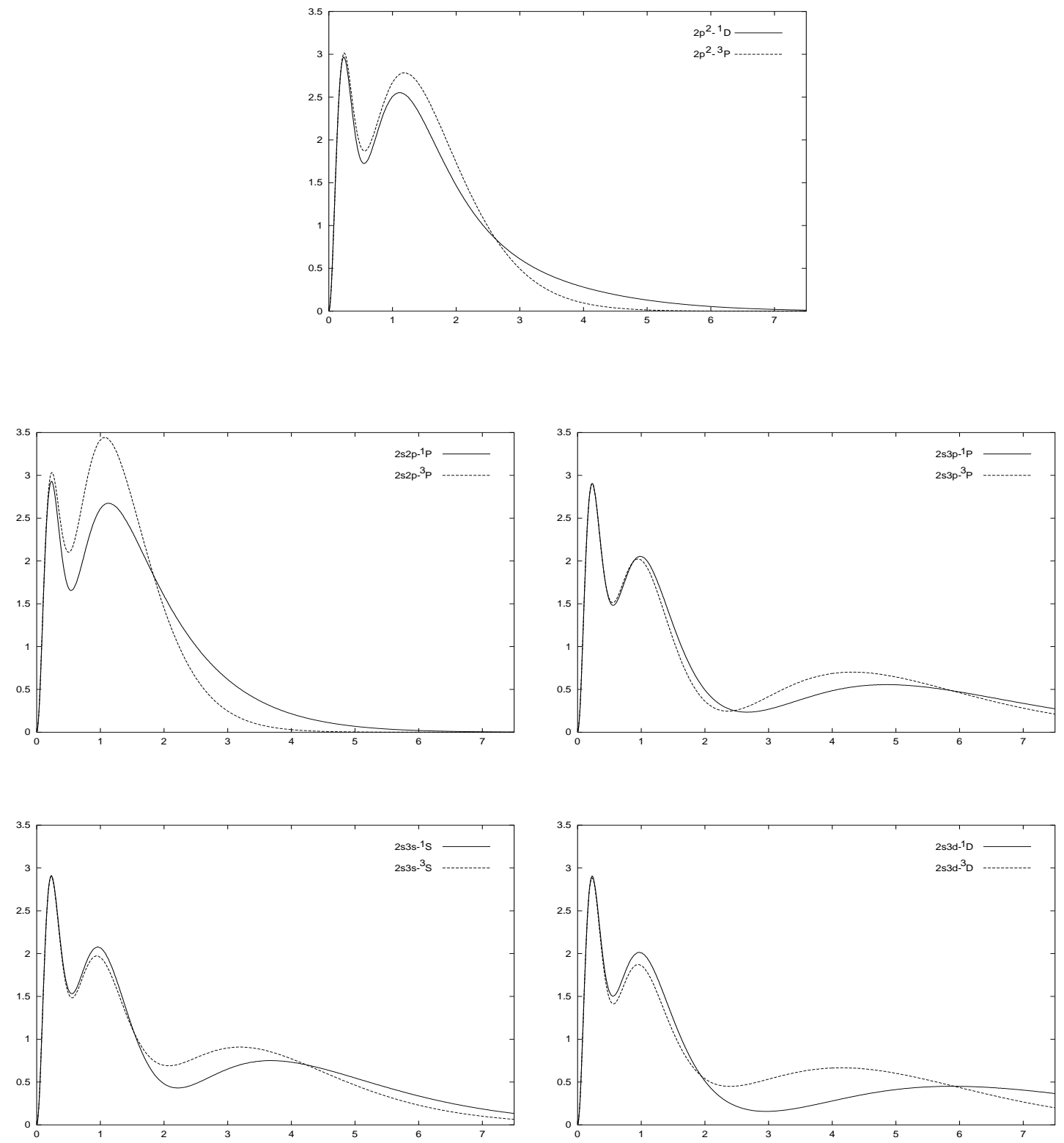

Figure 6 